\title{
Redshift of clusters and galaxies towards the Shapley Concentration ${ }^{\star}$
}

\author{
H. Quintana ${ }^{1, \star \star}$, J. Melnick ${ }^{2}$, D. Proust ${ }^{3}$, and L. Infante ${ }^{1}$ \\ 1 Departamento de Astronomía y Astrofísica, Pontificia Universidad Católica de Chile, Casilla 104, Santiago 22, Chile \\ 2 European Southern Observatory, Casilla 19001, Santiago 19, Chile \\ 3 Observatoire de Paris, Section de Meudon, DAEC, F-92195 Meudon Cedex, France
}

Received October 15; accepted December 19, 1996

\begin{abstract}
We report velocity measurements of galaxies in this, the densest mass concentration within $z=0.1$, obtained with the multifiber spectrograph MEFOS at ESO, La Silla. We derive redshifts of a number of Abell clusters and draw implications for the supercluster structure. The clusters A3554, A3566, A3577 and AS718 are confirmed as members, while the clusters A3524, A3531, A3542, A3545 and A3549 are shown to be background systems and A3581 a foreground one.
\end{abstract}

Key words: galaxies: clusters; distances and redshifts large-scale structure of the universe

\section{Introduction}

The Shapley Concentration or Supercluster (S.C) of galaxies, centered at A3558 and comprising many clusters and groups of galaxies in the approximate redshift range $z=0.04-0.055$ has been recognized as the densest, and one of the largest concentrations of visible matter within the $z<0.1$ Universe. Several efforts are underway to collect numerous velocities in the region to map the concentration and evaluate its dynamical state. The central cluster A3558 has been investigated by Bardelli et al. (1996) with a pointed ROSAT PSPC observation, leading to a luminosity $L_{x}=1.110^{44} \mathrm{~h}^{-2} \mathrm{erg} \mathrm{s}^{-1}$ in the energy range $[0.5-2.0] \mathrm{keV}$, within a radius of $0.8 \mathrm{~h}^{-1} \mathrm{Mpc}$ and a total mass $M_{\text {tot }}=3.110^{14} \mathrm{~h}^{-1} M_{\odot}$ within an Abell radius $1.5 \mathrm{~h}^{-1} \mathrm{Mpc}$. Another member of the Shapley S.C, A3266 has been extensively studied by Quintana et al. (1996) from observations carried out both at Las Campanas and Cerro Tololo observatories. A remarkable

\footnotetext{
Send offprint requests to: H. Quintana

* Based on observations made at ESO, La Silla, Chile.

$\star \star$ Presidential Chair in Science.
}

velocity substructure is found, interpreted as a tidal outgoing arm reaching from the center to the northernmost extensions surveyed; the dynamical cluster mass is estimated at about $510^{15} \mathrm{~h}_{50}^{-1} M_{\odot}$.

The complexity of the region implies that the Abell distance class is not a very good distance indicator (Quintana et al. 1995). In fact, clusters of Abell distance classes 3, 4 and 5 belong to the S.C. It is thus necessary to use redshifts to be able to evaluate the supercluster membership and extension. The membership of several clusters is not yet clear neither is the total extension of the supercluster. As part of our observational work in this area we used the MEFOS spectrograph on one observing session in April 1994. Given the wide field of the MEFOS instrument and its limitation on angular distance between objects, we obtained spectra of a number of galaxies located in the areas of 15 ACO (Abell et al. 1989) clusters that had no, or poorly determined, redshifts as reflected at the time of the observations.

In Sect. 2 we describe the observations and reductions. In Sect. 3 we present the new velocities and comparisons with published values and in Sect. 4 we discuss the results for the individual clusters. In Sect. 5 we summarize the implications of these results for the supercluster structure $\left(H_{0}=100 h \mathrm{~km} \mathrm{~s}^{-1} \mathrm{Mpc}^{-1}\right.$, is used throughout, with $h=0.5$ if needed). For comparison purposes we also observed one MEFOS field in A1060, and we also report those velocities here.

\section{Observations and reductions}

The spectroscopic observations were made on the nights of April 10-13 1994 using the MEFOS spectrograph on the ESO $3.6 \mathrm{~m}$ telescope. MEFOS is a robot controlled fiber instrument with $30 \mathrm{arms}$, disposed like fishermen around a pond, about the $1^{\circ}$ prime focus field. The detector is a Tek $512 \times 512$ pixels $^{2}$ CCD, ESO 32 . The dispersion on the CCD was $170 \AA / \mathrm{mm}$ and the spectra covered the range from 3800 to $5100 \AA$. The resolution given by 
the combination of $100 \mu \mathrm{m}$ fibers and CCD $15 \mu \mathrm{m}$ pixel with the standard Boller \& Chivens spectrograph on the $3.6 \mathrm{~m}$ telescope was $3.8 \AA$. The $100 \mu \mathrm{m}$ fibers are disposed in pairs, one for object and sky and one for sky. The arms bulk only permitted a minimum angular distance of $6^{\prime}$ between targets, a strong constraint. Switching between the fibers permits precise sky substraction. We exposed twice for 15-20 minutes. Further description of this equipment and discussion of its performance can be found in Felenbok et al. (1996).

The MEFOS system permits one to obtain a CCD image of a square field $30^{\prime \prime}$ in size around each target, providing a precise centering of the fibers. The original target selection was based on catalogs produced by the MAMA machine from scans of the R ESO/SRC survey plates done for our project (Slezak et al. 1996; Infante et al. 1996). Due to the rather low galactic latitude of the fields, some $25-30 \%$ of the galaxies identified by the MAMA software proved to be confused or merged objects. Such a rather high fraction is also related to the use of a glassplate copy on which the measured seeing on the stars can be evaluated around 3 arcsec; confusion mostly occured between a faint or low surface brightness galaxy and a nearby faint star. The MEFOS procedure allows the fiber to be re-centred when necessary. This possibility led to the observation of some faint galaxies, much fainter than the homogeneous sample originally intended. Moreover, due to the severe angular limitation referred above, we were prevented from observing many bright galaxies. Therefore, the observed galaxies are far from been a magnitude limited sample. In the galaxy selection process no consideration was taken of the few previously known redshifts, thus generating some overlap for comparison purposes.

The data reduction was carried out at Meudon using the standard IRAF package. This software package efficiently corrects for bias, flat field, and sky emission, combines the individual exposures and provides 1D spectra calibrated in wavelength. Wavelength calibrations were done using a 5 min. exposure with an $\mathrm{He}-\mathrm{Ar}$ or a $\mathrm{He}-\mathrm{Ne}$ lamp taken inmediately before and after each observation. The spectra were rebinned with a scale of $1 \AA /$ bin equally spaced in wavelength. The velocities were determined from the cross-correlation procedure with stellar and galaxy template spectra of known radial velocity, according to Tonry \& Davis (1979). We used the RVSAO package developped at Harvard (Kurtz et al. 1991; Mink et al. 1995).

\section{Velocities and comparisons}

In Table 1 we list positions, morphological types from Richter (1989) and heliocentric velocities for 12 individual galaxies in the cluster A1060, while in Table 2 we do that for 258 galaxies in 15 clusters in the direction of the
Shapley supercluster ${ }^{1}$. The columns give the following informations:

1. an identification number corresponding to the MEFOS arm used

2. right ascension (hour, min, sec)

3. declination (degree, minute, second)

4. estimated $R$ magnitude, from the MAMA scans

5. morphological type, deduced from a visual inspection either of the MEFOS images, or from the POSS

6 . heliocentric radial velocity

7. velocity errors

8. $R$ value of the correlation, from Tonry \& Davis (1979)

9. comments, including velocities from literature.

We emphasised the MAMA magnitudes have not been accurately calibrated, so these values are just a relative scale.

In order to test the external accuracy of our velocities, we compared our redshift measurements with data available in the literature. Furthermore, comparisons we made separately for the well-known A1060 galaxy cluster. We obtained respectively $\left\langle V_{0}-V_{\text {ref }}\right\rangle=14.9 \mathrm{~km} \mathrm{~s}^{-1}$, the standard deviation in the difference being $42 \mathrm{~km} \mathrm{~s}^{-1}$ (data from Richter 1989). All these results are consistent with the errors listed in Tables 1 and 2. The velocities in the present study agree with those previously published within the $2 \sigma$ level.

For completeness purposes we list in Table 3 the velocities available in the literature for all galaxies in the $1^{\circ}$ diameter fields observed ${ }^{2}$. These values are taken into account in the discussion below.

\section{Discussion}

The $1^{\circ}$ MEFOS fields span a diameter of $2.6 \mathrm{~h}^{-1} \mathrm{Mpc}$ at the Shapley supercluster redshift. Therefore, we expected to observe galaxies belonging both to the targeted Abell clusters and to the supercluster, besides some background/foreground galaxies in each MEFOS field. This is borne by the velocities reported in Table 2 . Velocity membership in the clusters studied in the present work show large differences from one cluster to another. Figure 1 shows the velocity histograms of the 15 clusters studied in the present paper.

Many galaxies are background and, in some fields, it is unclear whether an Abell cluster is present at all. In Table 3 we summarize the derived kinematical parameters for the clusters or clumps that can be identified in the area. Figure 2 plots cone diagrams of the observed

1 Tables 1, 2 and 3 are available in electronic form at the CDS via anonymous ftp to cdsarc.u-strasbg.fr (130.79.128.5) or via http://cdsweb.u-strasbg.fr/Abstract.html

2 This research has made use of the NASA/IPAC extragalactic database (NED) which is operated by the Jet Propulsion Laboratory, Caltech, under contract with the National Aeronautics and Space Administration. 

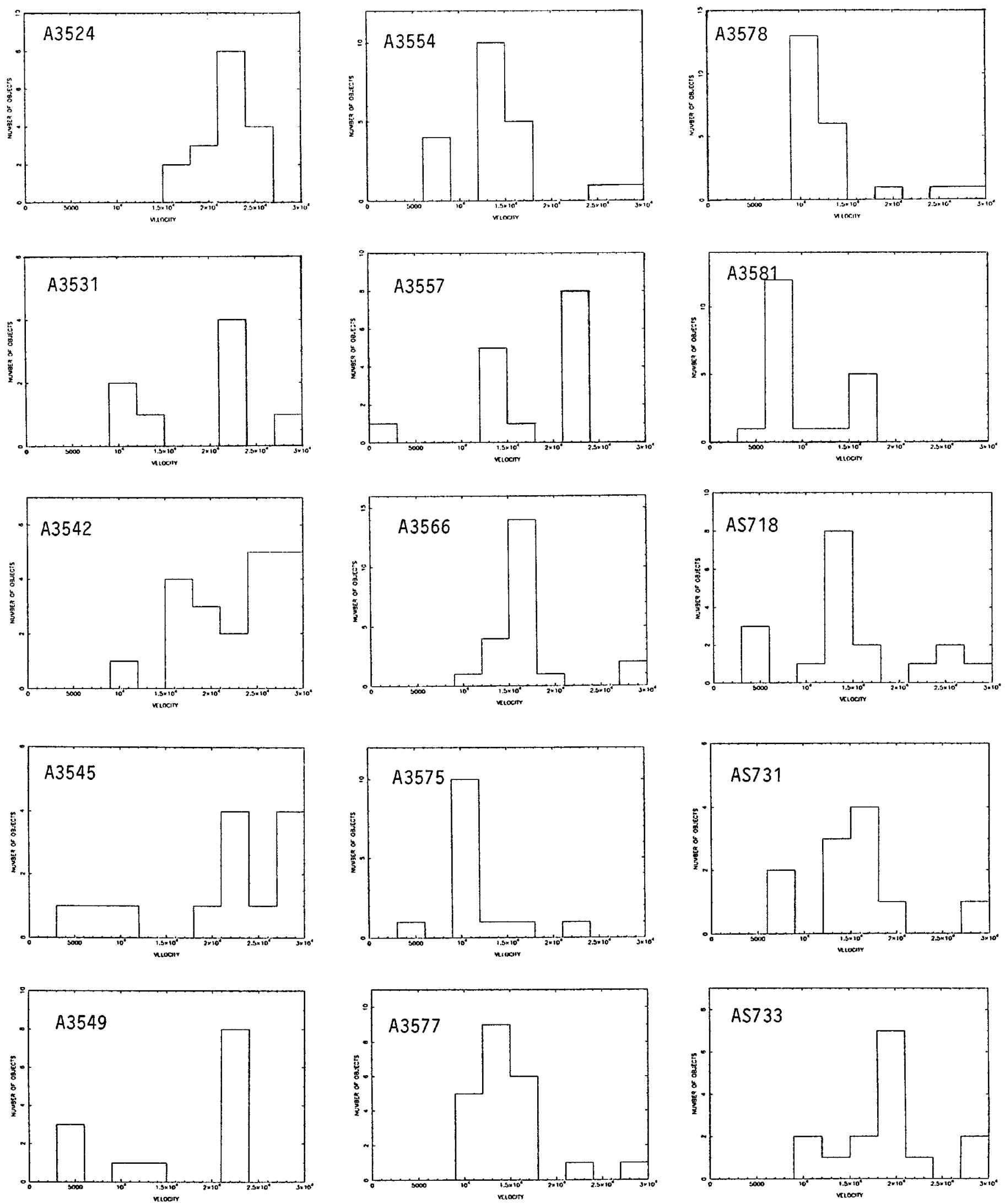

Fig. 1. Velocity histograms of 15 clusters from the present paper 
Table 4. Mean redshifts and dispersions in the observed clusters

\begin{tabular}{llllllllll}
\hline Cluster & component & BM & $\mathbf{D}, \mathbf{R}$ & $N_{\text {vel. }}$ & $z$ & $\Delta z$ & $\sigma_{V}$ & $\Delta \sigma_{V}$ & comments \\
\hline A3524 & & I & 0,5 & 14 & 0.0743 & 0.000103 & 1727 & 14 & cD not observed \\
A3531 & & II & 0,5 & 5 & 0.0749 & 0.000220 & 525 & 40 & \\
A3542 & a & I & 0,4 & 10 & 0.0919 & 0.000153 & 1275 & 17 & complex structure \\
& b & & & 7 & 0.1314 & 0.000150 & 2152 & 23 & \\
A3545 & I-II & 0,6 & 5 & 0.0742 & 0.000137 & 1276 & 23 & \\
A3549 & I-II & 1,5 & 8 & 0.0755 & 0.000170 & 715 & 24 & \\
A3554 & I-II & 1,4 & 16 & 0.0486 & 0.000137 & 404 & 23 & member of the SC \\
A3557 & a & I-II & 0,5 & 5 & 0.0476 & 0.000167 & 630 & 21 & complex structure \\
& b & & & 8 & 0.0770 & 0.000167 & 213 & 10 & \\
A3566 & & II & 2,4 & 18 & 0.0516 & 0.000123 & 567 & 13 & member of the SC \\
A3575 & II & 0,4 & 12 & 0.0375 & 0.000130 & 572 & 12 & foreground \\
A3577 & II & 2,4 & 17 & 0.0496 & 0.000143 & 495 & 21 & member of the SC \\
A3578 & I-II & 1,3 & 14 & 0.0372 & 0.000123 & 410 & 15 & foreground \\
A3581 & I & 0,3 & 18 & 0.0226 & 0.000134 & 517 & 16 & very foreground \\
AS718 & I & 0,4 & 8 & 0.0478 & 0.000133 & 418 & 13 & \\
AS731 & I-II & 0,4 & 7 & 0.0505 & 0.000233 & 657 & 26 & \\
AS733 & II & 0,5 & 7 & 0.0694 & 0.000190 & 551 & 30 & background \\
\hline
\end{tabular}

Right Ascension

Declination

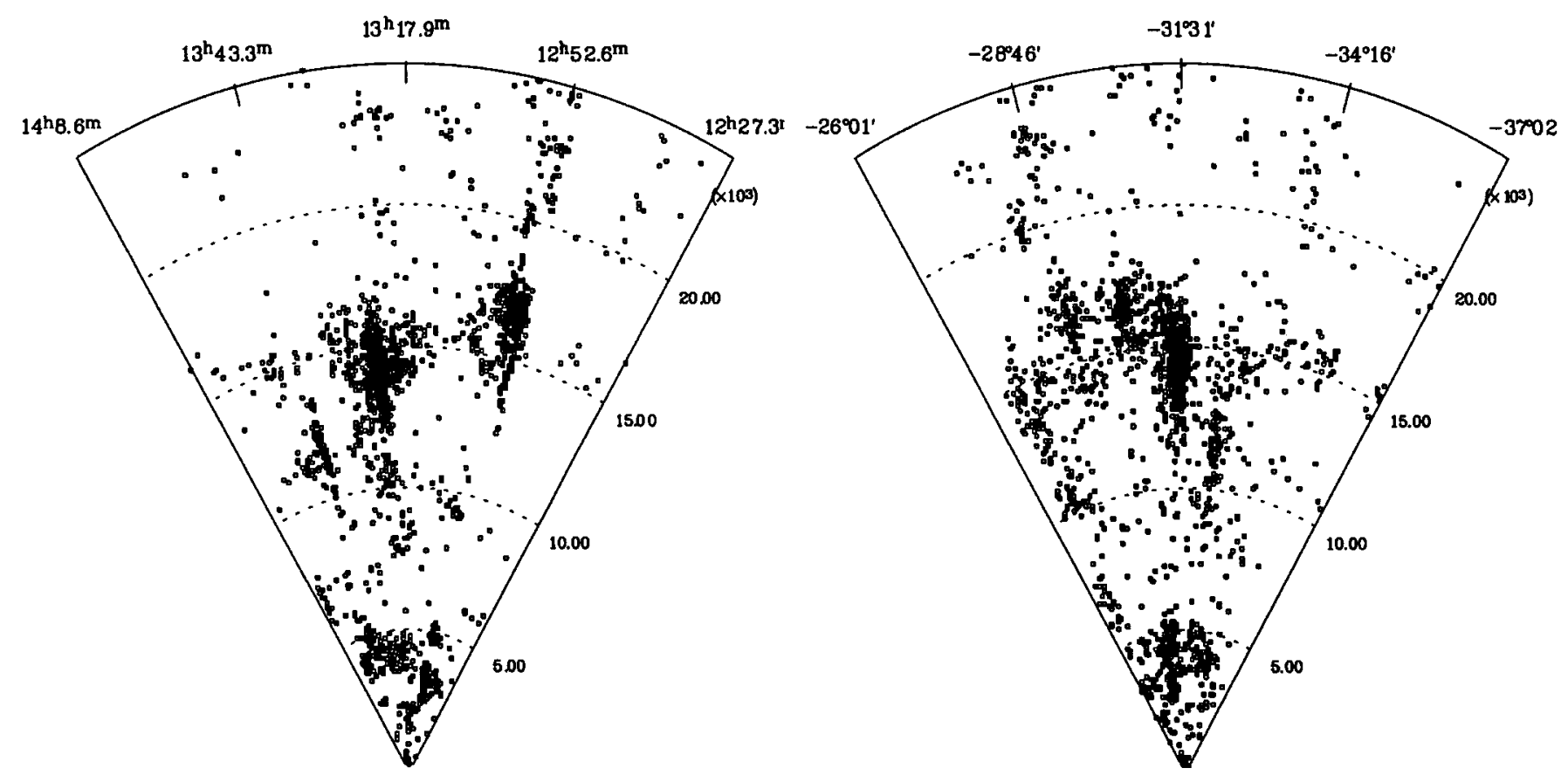

Fig. 2. Wedge diagram in right ascension (left) and declination (right) of galaxies with $c z \prec 25000 \mathrm{~km} \mathrm{~s}^{-1}$ in the central region of the Shapley supercluster. Data from the literature and from the present paper are displayed 
galaxies, plus all galaxies with known redshifts in the supercluster. Figure 3 shows the radial velocity histogram for the 1385 galaxies having data available in the literature and from the present work, with $c z<35000 \mathrm{~km} \mathrm{~s}^{-1}$ in the central region of the supercluster. There are two clear peaks in the distribution, one around $4223 \mathrm{~km} \mathrm{~s}^{-1}$ with $\sigma=580 \mathrm{~km} \mathrm{~s}^{-1}$ (208 objects), corresponding to galaxies in the general Hydra-Centaurus region, and the main peak centered at $14817 \mathrm{~km} \mathrm{~s}^{-1}$ with $\sigma=1150 \mathrm{~km} \mathrm{~s}^{-1}$ (620 galaxies), which corresponds to the Shapley supercluster. Note that the first peak can be related with the nearby cluster A3627 $\left(\bar{V}=4882 \mathrm{~km} \mathrm{~s}^{-1}, \sigma=\right.$ $903 \mathrm{~km} \mathrm{~s}^{-1}$ ) associated with the "Great Attractor" (Kraan-Korteweg et al. 1996). There may be a third peak near $11000 \mathrm{~km} \mathrm{~s}^{-1}$ possibly connected to the supercluster as shown on the wedge diagram displayed in Fig. 3. The two dimensional distribution of galaxies in the central region of the Shapley supercluster using different symbols for different velocity ranges (as indicated in the figure caption) is shown in Fig. 4.

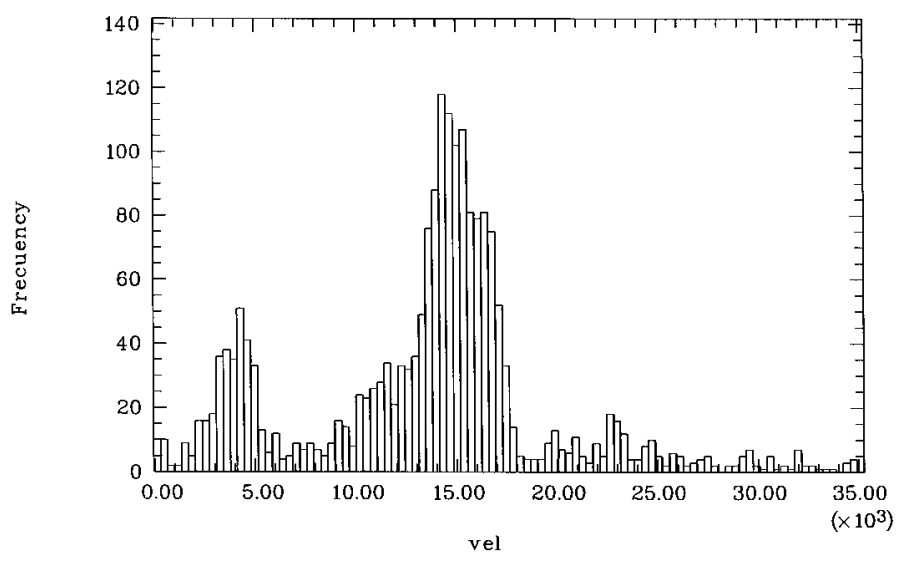

Fig. 3. Radial velocity histrogram for all the galaxies with $c z \prec 35000 \mathrm{~km} \mathrm{~s}^{-1}$ in the central region of the Shapley Supercluster

\section{Notes on individual clusters:}

We have inspected by eye the observed galaxies and surrounding fields on the film copies of the $J$ and $R$ SRC/ESO surveys to evaluate the relationship between the supercluster members. Integrating comments from the previous literature, we can make the following comments: A3524:

Background cluster with Bautz-Morgan type I. The central cD galaxy was not observed, but the 14 closest observed galaxies, with velocities in the range $19700-$ $25300 \mathrm{kms}^{-1}$, are consistent with being members of a cluster. This supersedes the value $z=0.054$ given by Quintana et al. (1995) taken from the NED database. Only 2 galaxies are members of the Shapley Concentration (S.C) and 4 are background at larger distances.

\section{A3531:}

Only one galaxy appears to be member of the S.C. Except 2 foreground galaxies, all the other objects are in a very large range of background velocities; the reality of the cluster itself is questionable. The average redshift quoted in Table 3 is derived from the five galaxies in the range $21600-23100 \mathrm{~km} \mathrm{~s}^{-1}$.

\section{A3542:}

The field in the direction of A3542 is complex. Postman \& Lauer (1995) identify galaxy No. 29 as the brightest cluster galaxy (BCG), with a velocity of $10395 \mathrm{kms}^{-1}$, in agreement with our measurement and with Vettolani et al. (1990). However, no other measured galaxy in the field has a similar redshift, making the BCG a likely spurious identification. Vettolani et al. (1990) suggested the reality of this cluster as a member of the S.C on the basis of 2 measured galaxies (as given in Table 3 ), with a redshift 0.051 . We measured two other galaxies in that same redshift range, i.e. Nos. 15 and 18. We also observed 8 galaxies in the range $26500-30000 \mathrm{~km} \mathrm{~s}^{-1}$, range in which Vettolani et al. (1990) also observed two galaxies between $26500-27000 \mathrm{~km} \mathrm{~s}^{-1}$. Furthermore, we obtained 4 galaxies in the range $19000-21100 \mathrm{~km} \mathrm{~s}^{-1}$ and 7 more in the far background. We can only conclude to the presence of a superposition of at least 4 sheets of galaxies: at the S.C. redshift, at $20000 \mathrm{~km} \mathrm{~s}^{-1}$ and two groups in the ranges $26000-30000 \mathrm{~km} \mathrm{~s}^{-1}$ (10 objects) and $36500-42000 \mathrm{~km} \mathrm{~s}^{-1}$ (7 objects), the later with dispersion velocities respectively $\sigma=1275$ and $2152 \mathrm{~km} \mathrm{~s}^{-1}$.

\section{A3545 and A3549:}

These two areas contain only one galaxy member of the S.C. A possible cluster with 13 objects is defined in the range $20700-23700 \mathrm{kms}^{-1}$ with a mean value $\bar{V}=22482 \mathrm{kms}^{-1}$ and a dispersion velocity about $938 \mathrm{~km} \mathrm{~s}^{-1}$. Two sets of background galaxies are also located around 28000 and $40000 \mathrm{~km} \mathrm{~s}^{-1}$.

\section{A3554:}

This cluster clearly belongs to the S.C. 15 galaxies have been observed in the range $14000-15500 \mathrm{~km} \mathrm{~s}^{-1}$ with a mean value $\bar{V}=14585 \mathrm{~km} \mathrm{~s}^{-1}$ and a dispersion velocity about $412 \mathrm{~km} \mathrm{~s}^{-1}$.

A3557:

6 galaxies have velocities consistent with the S.C. A background structure with 8 objects appears in the range $22000-23500 \mathrm{~km} \mathrm{~s}^{-1}$ with a mean value $\bar{V}=23065 \mathrm{kms}^{-1}$ and a dispersion velocity about $302 \mathrm{~km} \mathrm{~s}^{-1}$, similarly to A3545 and A3549. 5 galaxies are in the range $31000-35000 \mathrm{~km} \mathrm{~s}^{-1}$.

\section{A3566:}

This cluster belongs to the S.C. 18 galaxies (14 from the present work and 4 in Vettolani et al. 1990) are in the range $14500-16500 \mathrm{~km} \mathrm{~s}^{-1}$ with a mean velocity $\bar{V}=15463 \mathrm{~km} \mathrm{~s}^{-1}$ and a dispersion velocity of $567 \mathrm{~km} \mathrm{~s}^{-1}$. 

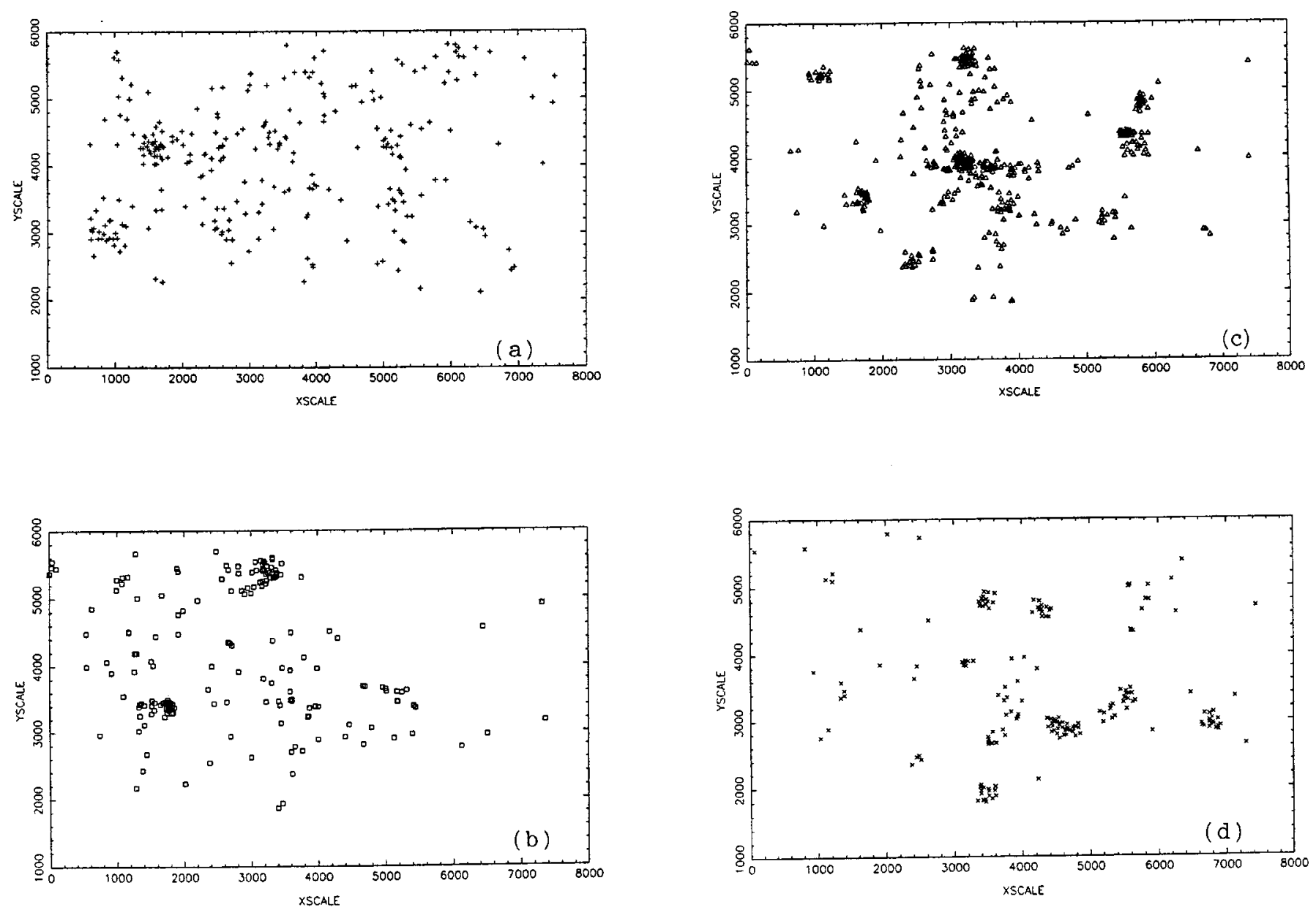

Fig. 4. The two dimensional distribution of galaxies in the central region of the Shapley supercluster. Four different velocity cuts have been represented by different symbols. Plusses: $v \prec 6000 \mathrm{~km} \mathrm{~s}^{-1}$ a); Open squares: $6000 \prec v \prec 12000 \mathrm{~km} \mathrm{~s} \mathbf{b}^{-1} \mathbf{b}$; Open triangles: $12000 \prec v \prec 18000 \mathrm{~km} \mathrm{~s}^{-1} \mathbf{c}$ ), and Crosses: $v \succ 18000 \mathrm{~km} \mathrm{~s}^{-1} \mathbf{d}$ ). The $X$ and $Y$ scale are given in arcsec from the center of the figure with R.A: $13 \mathrm{~h} 15 \mathrm{mn}$ and Dec: $-31^{\circ} .00$

\section{A3575, A3577 and A3578:}

These three clusters have a common foreground structure defined by 26 galaxies at a mean velocity of $11199 \mathrm{~km} \mathrm{~s}^{-1}$ with a dispersion about only $400 \mathrm{~km} \mathrm{~s}^{-1}$. The S.C. reaches these positions as 20 galaxies are members of the S.C. with a mean value $\bar{V}=13961 \mathrm{~km} \mathrm{~s}^{-1}$ with $\sigma=692 \mathrm{~km} \mathrm{~s}^{-1}$ while only 20 galaxies are background ones. Note that for A3578 Vettolani et al. (1990) obtained two similar sets of velocities only based upon 5 velocities. This foreground structure has a similar redshift to A3571 at $z=0.040$ (Quintana \& de Souza 1993). Further data can clarify the question of the conecction of this structure to the S.C.

\section{A3581:}

This cluster is really a foreground one with 13 significant members and five from the litterature (see Vettolani et al. 1990), in the range $5900-7900 \mathrm{~km} \mathrm{~s}^{-1}$ with $\bar{V}=6771 \mathrm{~km} \mathrm{~s}^{-1}$ and a dispersion velocity about $516 \mathrm{~km} \mathrm{~s}^{-1}$.

\section{AS718, AS731 and AS733:}

8 galaxies from AS718 belong to the S.C $\left(\bar{V}=14339 \mathrm{~km} \mathrm{~s}^{-1}\right.$, dispersion $\left.418 \mathrm{~km} \mathrm{~s}^{-1}\right)$, and 3 objects are foreground ones. 7 galaxies from AS731 are members of the S.C $\left(\bar{V}=15146 \mathrm{~km} \mathrm{~s}^{-1}\right.$, dispersion $\left.657 \mathrm{~km} \mathrm{~s}^{-1}\right)$, while a background group with 7 galaxies appears around $20000 \mathrm{~km} \mathrm{~s}^{-1}$ in AS733.

\section{Conclusions}

Preliminary conclusions from this study are the following:

1. We have confirmed that 4 clusters (A3554, A3566, AS718, AS731) are members of the supercluster. We have also found that one cluster, A3524, originally reported to be a member of the S.C., is really background.

2. We have shown that 4 clusters are formed by a superposition of several layers or sheets of galaxies, one of them located at the general S.C. redshift range. A3577 
has a second component at $z \simeq 0.04$, together with A3571, A3575 and A3578. The clusters A3542, A3557, and AS733 have a second background component.

3. We have shown that, at least, 4 clusters (A3571, A3575, a component of A3577 and A3578) located in the R.A. and dec zones $13 \mathrm{~h} 30-14 \mathrm{~h} 15$ are part of a structure located in front or extending towards the front of the S.C. A more precise definition of a possible connection with the S.C. has to await more data.

4. The cluster A3581 is a foreground system. Further to A3524, A3545 and A3549 are also background clusters.

5. Walls at $z \simeq 0.066$ and $z \simeq 0.092$ seem to be present behind wide zones of the S.C.

Acknowledgements. We are grateful to ESO for this unique opportunity to use the MEFOS facility. This research was partially supported by the cooperative program ECOS/CONICYT U9302. D.P particularly thanks the finantial support provided by ECOS and CONICYT during his stay at the P. Universidad Católica de Chile. H.Q gratefully acknowledges partial support from FONDECYT Grant $\mathrm{N}^{\circ} 1960413$ and the award of a 1995 Presidential Chair in Science. We also thank our referee, Dr. A. Fairall.

\section{References}

Abell G.O., Corwin H.G., Olowin R.P., 1989, ApJS 70, 1 Allen D.A., Norris R.P., Meadows V.S., Roche P.F., 1991, MNRAS 248, 258
Bade N., Fink H.H., Engels D., et al., 1995, A\&AS 110, 469

Bardelli S., Zucca E., Malizia A., et al., 1996, A\&A 305, 435

Danese L., De Zotti G., di Tullio G., 1980, A\&A 82, 322

Fairall A., Jones A., 1991, Southern Redshifts Catalogue, Publ. Dept. Astr. Univ. Cape Town

Felenbok P., Guerin J., Fernandez A., et al., 1996 (submitted to $A \& A$ )

Infante L., Slezak E., Quintana H., 1996, A\&AS (in press)

Kraan-Korteweg R.C., Woudt P.A., Cayatte V., et al., 1996, Nat 379, 519

Kurtz M.J., Mink D.J., Wyatt W.F., et al., 1991, ASP Conf. Ser. 25, 432

Lauberts A., Valentijn E.A., 1989, The Surface Photometry Catalogue of the ESO-Uppsala Galaxies, ESO

Mathews L.D., Gallagher J.S., Littleton J.E., 1995, AJ 110, 581

Mink D.J., Wyatt W.F., 1995, ASP Conf. Ser. 77, 496

Postman M., Lauer T.R., 1995, ApJ 440, 28

Quintana H., de Souza R., 1993, A\&AS 101, 475

Quintana H., Ramirez A., Melnick J., Raychaudhury S., Slezak E., 1995, AJ 110, 463

Quintana H., Ramirez A., Way M.J., 1996, AJ 112, 36

Richter O.G., 1989, A\&AS 77, 737

Slezak E., Infante L., Quintana H., 1996, A\&AS (in press)

Strauss M., Huchra J.P., Davis M., et al., 1992, ApJS 83, 29

Tonry J., Davis M., 1979, AJ 84, 1511

Vettolani G., Chincarini G., Scaramella R., Zamorani G., 1990, AJ 99, 1709 